\title{
Serum laminin levels in eosinophilic and non-eosinophilic chronic obstructive pulmonary disease patients
}

\author{
Gulay Dasdemir Ilkhan ${ }^{1, *} \odot$, Hakan Celikhisar ${ }^{2} \odot$
}

${ }^{1}$ Tire Public Hospital, Department of Chest Diseases, Tire, Izmir, Turkey ${ }^{2}$ Esrefpasa Metropolitan Municipality Hospital, Department of Chest Diseases, Izmir, Turkey

\section{*Correspondence}

gdasdemir1111@gmail.com

(Gulay Dasdemir Ilkhan)

\begin{abstract}
Aim: To compare serum laminin levels in eosinophilic and non-eosinophilic (neutrophilic) COPD patients and to define its association with disease severity.

Material and Method: This prospective study included patients with mild, moderate, severe, and very severe stable COPD and a control group of patients with a history of smoking but with no signs or symptoms of COPD. Spirometric measurements and Global Initiative for Chronic Obstructive Lung Disease (GOLD) criteria, was used to define the disease severity. Blood eosinophil percentage was recorded from complete blood counts. Serum laminin levels were measured in all patients.

Results: A total of 216 patients were included in the study. Ninety were in the eosinophilic COPD, 90 were in the non-eosinophilic COPD and 36 were in the control groups. In both COPD groups, serum laminin levels were significantly higher than in the control group $(P=0.001)$. In the eosinophilic COPD group, serum laminin levels were significantly higher than the non-eosinophilic COPD group $(P=0.001)$. With an increase in COPD severity, laminin levels were higher in both COPD groups $(P=0.001)$. In correlation analysis performed in all COPD patients, laminin levels were positively correlated with eosinophilia percentage $(\mathrm{r}=0.316, P=0.001)$ and negatively correlated with the FEV1/FVC ratio $(\mathrm{r}=-0.160, P=0.032)$.

Conclusion: Laminin has an important role in eosinophilic COPD and increased serum laminin levels are associated with an increase in serum eosinophilia percentage and a decrease in respiratory capacity.
\end{abstract}

\section{Keywords}

COPD severity; Eosinophilic COPD; Laminin; Non-eosinophilic-COPD

\section{Introduction}

Chronic Obstructive Pulmonary Disease (COPD) is one of the most common chronic diseases worldwide, causing severe morbidity and mortality [1]. Cigarette smoking is a welldefined risk factor in the development of COPD. In general, the main pathophysiological mechanism of COPD is the activation of pro-inflammatory cascades and neutrophilic inflammation resulting in irreversible airway obstruction [2]. However, in a subset of COPD patients, eosinophilic inflammation in the airways is defined as a distinct feature of this subtype [3, 4]. Previous studies have shown that an increase in the blood eosinophil count was associated with a risk of exacerbations, mortality, and decline in forced expiratory volume in 1 second (FEV1) in COPD patients [5, 6].

Extracellular matrix (ECM) components are known to promote cell proliferation, cell cycle progression, migration, and adhesion in airway smooth muscle cells in patients with COPD $[7,8]$. Alterations in ECM components are well-known characteristic features of COPD, providing airway wall remodeling and airway wall thickening [9]. ECM components also play an important role in the recovery of the epithelium following lung injury [10].

Laminin is an extracellular matrix component such as fibronectin and collagen and plays important roles in cell spreading promotion and migration. In persistent lung injury, the role of ECM components is important, especially in the repair processes [11]. Laminin is the primary component of basement membranes and is essential for cell behavior in the bronchiolar epithelium of COPD patients [12, 13].

In this study, we compared serum laminin levels in eosinophilic and non-eosinophilic (neutrophilic) COPD patients. Our primary objective was to determine the role of this ECM component in COPD subtypes and to define its association with disease severity. To the best of our knowledge, this is the first study that evaluated serum laminin levels in eosinophilic COPD patients.

\section{Material and method}

This prospective study included patients with mild, moderate, severe, and very severe stable COPD who presented to the 
TA B L E 1. Demographic features of study participants

Eosinophilic COPD (n: 90) Non-eosinophilic COPD (n: 90) Control group (n: 36) P

\begin{tabular}{|c|c|c|c|c|}
\hline Age (years) & $59.48 \pm 9.36$ & $60.11 \pm 9.07$ & $57.97 \pm 6.63$ & 0.47 \\
\hline Gender (F/M) & $20 / 70$ & $18 / 72$ & $7 / 29$ & 0.91 \\
\hline $\begin{array}{l}\text { Smoking history (Quit/still } \\
\text { smoking) }\end{array}$ & $28 / 62$ & $31 / 59$ & $17 / 19$ & 0.24 \\
\hline Pocket-year smoking & $37.58 \pm 14.85$ & $36.53 \pm 14.16$ & $33.05 \pm 12.55$ & 0.27 \\
\hline BMI $\left(\mathrm{kg} / \mathrm{m}^{2}\right)$ & $25.12 \pm 4.22$ & $24.28 \pm 4.32$ & $24.95 \pm 4.50$ & 0.40 \\
\hline
\end{tabular}

F: female, M: male, BMI: Body mass index.

pulmonology outpatient clinic between September 2019 and February 2020 at two centers. Patients with asthma, malignancy, psychiatric disorders, or chronic inflammatory diseases and patients receiving corticosteroid therapy were excluded. A control group was included of patients with a history of smoking but with no signs or symptoms of COPD and without eosinophilia. Blood eosinophil level of 3\% and above were used to define eosinophilia. Consecutive patients who agreed to participate were included in the study. The study was approved by the local ethics committee; Bezmialem Vakıf University Non-interventional Research Ethics Committee (2011KAEK-25 2019/12-08).

Demographic data, body mass index (BMI), smoking history and COPD exacerbations in the past year were recorded. BMI was calculated as body weight $(\mathrm{kg}) / \mathrm{height}^{2}\left(\mathrm{~m}^{2}\right)$. Spirometric measurements (FEV1 and FVC) and Global Initiative for Chronic Obstructive Lung Disease (GOLD) criteria were used to define the disease severity as follows stage one to four $[14,15]$.

Blood eosinophil percentage was recorded from complete blood count results. Serum laminin levels were measured using standard techniques. Blood was transferred to a yellow biochemistry tube, centrifuged, and the supernatant was taken to a Eppendorf tube and stored at -80 degrees. Serum laminin levels were determined by commercial kits using the ELISA (enzyme-linked immunosorbent test) method.

\subsection{Statistical analyses}

Statistical analyses were performed by using SPSS program v.21 (SPSS Inc., Chicago, IL). Continuous data were expressed as mean or median \pm standard deviation and categorical data were expressed as percentages. Chi-square test was used for the comparison of categorical data. Continuous data of two groups were compared with the student's $t$-test and continuous data of three groups were compared with the oneway ANOVA. Pearson's correlation analysis was performed to determine the association of different test parameters. A value of $P<0.05$ was considered statistically significant.

\section{Results}

A total of 216 patients were included in the study with 90 in eosinophilic COPD, 90 in non-eosinophilic COPD and 36 in the control groups. The demographic features of the study participants are summarized in Table 1. There was not any significant difference between the three groups regarding age, gender, smoking history, or BMI values.

Duration of COPD, COPD severity regarding spirometry findings and GOLD classification, and spirometry findings in COPD patients are summarized in Table 2. There was not any significant difference between the two COPD groups, regarding the duration or severity of COPD and spirometry findings.

Serum laminin levels are compared between the three groups (Table 3). In both COPD groups, serum laminin levels were significantly higher than the control group patients $(P<0.001)$; moreover in the eosinophilic COPD group, serum laminin levels were significantly higher than the non-eosinophilic COPD group $(P<0.001)$.

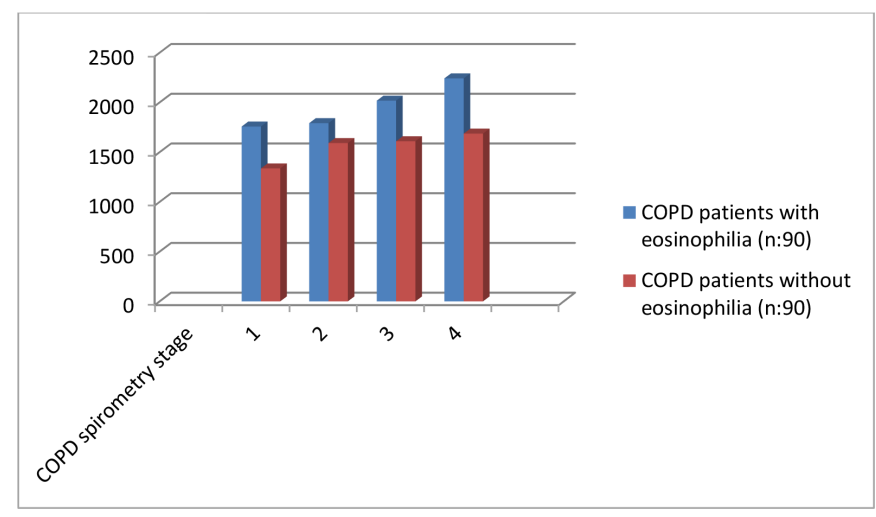

FIGURE 1. Distribution of serum laminin levels in COPD patients with or without eosinophilia at different stages of disease severity.

Serum laminin levels in COPD patients with different severity are compared in Table 4. With an increase in COPD severity, laminin levels were increasing significantly in both COPD groups (Fig. 1).

In correlation analysis performed in all COPD patients, laminin levels were positively correlated with eosinophilia percentage and negatively correlated with the FEV1/FVC ratio (Table 5).

\section{Discussion}

In this study, we determined that serum laminin levels were significantly higher in COPD patients, compared with sex- and age-matched control cases. Serum laminin levels were also significantly higher in the eosinophilic than the non-eosinophilic COPD groups. With an increase in 
TA B L E 2. Duration and severity of COPD and spirometry findings

\begin{tabular}{|lccc|}
\hline COPD duration (years) & Eosinophilic COPD (n: 90) & Non-eosinophilic COPD (n: 90) & $\boldsymbol{P}$ \\
\hline GOLD Stage (Predicted FEV1\%) & $8.01 \pm 2.88$ & $7.61 \pm 2.05$ & 0.59 \\
\hline $\mathbf{1 + 2}$ & & & \\
\hline $\mathbf{3 + 4}$ & $20+31$ & $25+14$ & 1.0 \\
\hline GOLD stage (Clinical) & $20+31$ & $25+14$ & \\
\hline A +C & & & 0.92 \\
\hline C + D & $22+21$ & $22+25$ & \\
\hline FEV1\% & $24+22$ & $21+23$ & 0.85 \\
\hline FVC\% & $61.73 \pm 21.32$ & $62.34 \pm 20.96$ & 0.63 \\
\hline FEV1/FVC\% & $70.93 \pm 19.25$ & $69.62 \pm 17.55$ & 0.80 \\
\hline
\end{tabular}

TA B L E 3. Serum laminin levels in study groups

\begin{tabular}{lcccc} 
& Eosinophilic COPD (n: 90) & Non-eosinophilic COPD (n: 90) & Control group(n: 36) & $P$ \\
Serum laminin levels & $1883.07 \pm 505.18$ & $1526.45 \pm 793.64$ & $861.52 \pm 706.16$ & 0.001 \\
\hline
\end{tabular}

TA B L E 4. Laminin levels in COPD patients with different severity

\begin{tabular}{|c|c|c|c|}
\hline & \multicolumn{2}{|c|}{ Eosinophilic COPD (n: 90) Non-eosinophilic COPD (n: 90) } & \multirow[t]{2}{*}{$\mathrm{P} 2$} \\
\hline GO & & & \\
\hline 1 & $1758.00 \pm 383.85$ & $1337.40 \pm 345.04$ & 0.001 \\
\hline 2 & $1792.41 \pm 406.20$ & $1592.67 \pm 482.62$ & 0.001 \\
\hline 3 & $2018.04 \pm 473.55$ & $1610.04 \pm 457.29$ & 0.001 \\
\hline 4 & $2242.92 \pm 572.20$ & $1687.26 \pm 438.07$ & 0.001 \\
\hline P1 & 0.001 & 0.001 & \\
\hline \multicolumn{4}{|c|}{ GOLD stage (Clinical) } \\
\hline A & $1627.27 \pm 326.53$ & $1426.12 \pm 481.85$ & 0.001 \\
\hline B & $1691.85 \pm 416.70$ & $1487.86 \pm 405.75$ & 0.001 \\
\hline C & $1991.72 \pm 355.77$ & $1578.19 \pm 493.52$ & 0.001 \\
\hline D & $2173.20 \pm 473.92$ & $1629.52 \pm 468.24$ & 0.001 \\
\hline P1 & 0.001 & 0.001 & \\
\hline
\end{tabular}

P1: Comparison between the eosinophilic COPD patients at different stages of severity; P2: Comparison between the eosinophilic and non-eosinophilic COPD patients

COPD severity, laminin levels increased significantly in both the eosinophilic and non-eosinophilic COPD groups. Finally, serum laminin levels were positively correlated with eosinophilia percentage and negatively correlated with the FEV1/FVC ratio in COPD patients.

The role of ECM components in the pathogenesis of COPD has been investigated in previous studies. In COPD development, multiple changes in the large and small airways, lung parenchyma, and pulmonary vascular structures are described. Hyperplasia and hypertrophy of airway smooth muscle cells and the accumulation of ECM components are the cause of thickened airways in COPD patients. Laminin is an ECM component that is increased in the sera of COPD patients [13]. Data about serum laminin levels in COPD patients is limited and, to our knowledge, this is the first study that compared serum laminin levels in eosinophilic and noneosinophilic COPD patients. In COPD, the main pathogenic
TA B L E 5. Correlation analysis performed between serum laminin levels and clinical findings in COPD patients

\begin{tabular}{lcc} 
& $\mathbf{r}$ & $\boldsymbol{P}$ \\
Eosinophilia percentage & $\mathbf{0 . 3 1 6}$ & $\mathbf{0 . 0 0 1}$ \\
\hline Age & 0.027 & 0.697 \\
\hline Pocket-years & 0.045 & 0.508 \\
\hline BMI & 0.054 & 0.43 \\
\hline COPD duration & 0.035 & 0.643 \\
\hline FEV1/FVC & $\mathbf{- 0 . 1 6}$ & $\mathbf{0 . 0 3 2}$ \\
\hline
\end{tabular}

$r$ : correlation coefficient, $P$ : significance level.

mechanisms are the remodeling of airways and blood vessels, and ECM components are known to play important roles in 
this process. Kranenburg et al. [16] showed increased laminin beta2 expression in airway smooth muscle in patients with COPD which was inversely correlated with the FEV1 values. Prabhla et al. [17] reported a prominent role for laminin in airway smooth muscle function and in inflammation, airway hyperresponsiveness, and remodeling in asthma. Reticular basement membrane thickening occurs both in asthma and COPD but ECM components such as collagen I and laminin were stained significantly stronger in asthma than in COPD [18]. In this study, in keeping with the previous literature, we also report higher serum laminin levels in COPD patients compared with controls which inversely correlated with the FEV1/FVC ratio.

The predominant cell types in COPD are the neutrophils and alveolar macrophages with eosinophilic inflammation most often associated with asthma. However, elevated eosinophil counts in sputum or serum are reported in COPD patients, especially during exacerbations. The use of peripheral blood cell counts is more simple and inexpensive than sputum investigations and in asthma patients, the accuracy of both blood eosinophils and sputum eosinophilia have been demonstrated [19]. Though the role of eosinophilia in COPD is still not understood they are thought as the regulator of remodeling in COPD [20, 21]. Eosinophils store cytotoxic basic proteins in secondary granules and play important roles in the production of reactive oxygen species and cytokines which are important in airway epithelial cell damage. For that reason, eosinophils are increased in airway inflammation during acute COPD exacerbations [22-24]. Singh et al. [4] reported that, in the ECLIPSE (Evaluation of COPD Longitudinally to Identify Predictive Surrogate End points) cohort, patients with persistent eosinophilia ( $\geq 2 \%$ ) were older, predominantly male with milder disease and having fewer symptoms and without significant lung function decline. On the other hand, Casanova et al also reported that in patients with COPD, blood eosinophils $\geq 300$ cells $\cdot \mu \mathrm{L}^{-1}$ persisting over 2 years was not a risk factor for COPD exacerbations and high eosinophil count was associated with better survival [25].

Though eosinophilia was associated with COPD exacerbations, all patents in our study had stable disease with disease severity that was similar between eosinophilic and non-eosinophilic COPD groups. Moreover, in eosinophilic COPD, which is regarded as a COPD subtype having more similar pathogenesis with asthma, we determined significantly higher serum laminin levels compared with the neutrophilic COPD. Serum laminin levels were also correlated with the eosinophilia percentage, suggesting the role of laminin in eosinophilic COPD.

There are some limitations to this study that need to be discussed. We did not ask for the medications the patients were using and corticosteroids are known to cause alterations in blood eosinophilia. Secondly, a single measurement may not be accurate to identify patients with eosinophilia, and investigating persistent eosinophilia in COPD patients may have provided additional data. However, since all our patents were stable without exacerbations, these data are likely to be meaningful.

In conclusion, for the first time in the literature, we report significantly higher serum laminin levels in eosinophilic than the non-eosinophilic COPD patients. Serum laminin levels were positively correlated with eosinophilia percentage and negatively correlated with the FEV1/FVC ratio in COPD patients. Regarding postulate that laminin plays an important role in eosinophilic COPD and increased serum laminin levels are associated with an increase in serum eosinophilia percentage and a decrease in respiratory capacity. Further studies investigating the role of laminin in the pathogenesis of COPD are needed to characterize new treatment modalities.

\section{AUTHOR CONTRIBUTIONS}

Dasdemir Ilkhan Gulay: Processing, preparation and writing of the collected information, critically and intellectually evaluating the content of the article, analysis of the content of the article. Celikhisar Hakan: Literature search and analysis, design and concept of the article, case collection, data gathering and workflow planning, forming the general lines and framework of the study. Arslan Seray: Evaluating the accuracy of the data, statistical evaluation of the article data and the arrangement and interpretation of these data, regulation of the presentation of the information and data in the article.

\section{ETHICS APPROVAL AND CONSENT TO PARTICIPATE}

The study was approved by the local ethics committee; Bezmialem Vakıf University Non-interventional Research Ethics Committee. (2011- KAEK-25 2019/12-08).

\section{ACKNOWLEDGMENT}

Thanks to all the peer reviewers for their opinions and suggestions.

\section{FUNDING}

The authors declared that this study has received no financial support.

\section{CONFLICT OF INTEREST}

No conflict of interest was declared by the authors.

\section{REFERENCES}

[1] Crisafulli E, Torres A. COPD 2017: a year in review. Journal of Chronic Obstructive Pulmonary Disease. 2018; 15: 118-122.

[2] Van Eeden SF, Sin DD. Oxidative stress in chronic obstructive pulmonary disease: a lung and systemic process. Canadian Respiratory Journal. 2013; 20: 27-29.

[3] Tworek D, Antczak A. Eosinophilic COPD - a distinct phenotype of the disease. Advances in Respiratory Medicine. 2017; 85: 271-276.

[4] Singh D, Kolsum U, Brightling CE, Locantore N, Agusti A, Tal-Singer $\mathrm{R}$, et al. Eosinophilic inflammation in COPD: prevalence and clinical characteristics. European Respiratory Journal. 2014; 44: 1697-1700.

[5] Bafadhel M, Pavord ID, Russell REK. Eosinophils in COPD: just another biomarker? The Lancet Respiratory Medicine. 2017; 5: 747-759.

[6] Bafadhel M, McKenna S, Terry S, Mistry V, Reid C, Haldar P, et al. Acute exacerbations of chronic obstructive pulmonary disease: identification of 
biologic clusters and their biomarkers. American Journal of Respiratory and Critical Care Medicine. 2011; 184: 662-671.

[7] Dekkers BGJ, Schaafsma D, Nelemans SA, Zaagsma J, Meurs H. Extracellular matrix proteins differentially regulate airway smooth muscle phenotype and function. American Journal of Physiology-Lung Cellular and Molecular Physiology. 2007; 292: L1405-L1413.

[8] Wang Z, Li R, Zhong R. Extracellular matrix promotes proliferation, migration and adhesion of airway smooth muscle cells in a rat model of chronic obstructive pulmonary disease via upregulation of the PI3K/AKT signaling pathway. Molecular Medicine Reports. 2018; 18: 3143-3152.

[9] Johnson PR, Black JL, Carlin S, Ge Q, Underwood PA. The production of extracellular matrix proteins by human passively sensitized airway smoothmuscle cells in culture: the effect of beclomethasone. American Journal of Respiratory and Critical Care Medicine. 2000; 162: 2145-2151.

[10] White SR, Dorscheid DR, Rabe KF, Wojcik KR, Hamann KJ. Role of very late adhesion integrins in mediating repair of human airway epithelial cell monolayers after mechanical injury. American Journal of Respiratory Cell and Molecular Biology. 1999; 20: 787-796.

[11] Crosby LM, Waters CM. Epithelial repair mechanisms in the lung. American Journal of Physiology-Lung Cellular and Molecular Physiology. 2010; 298: L715-L731.

[12] Parameswaran K, Willems-Widyastuti A, Alagappan VKT, Radford K, Kranenburg AR, Sharma HS. Role of extracellular matrix and its regulators in human airway smooth muscle biology. Cell Biochemistry and Biophysics. 2006; 44: 139-146.

[13] Johansson O, Erjefält J, Bjermer L, Löfdahl C-G, Westergren-Thorsson $\mathrm{G}$, Hallgren O. Aberrant intracellular expression of laminin $\alpha-2$ and -5 in bronchiolar epithelium of COPD patients. European Respiratory Journal. 2014; 14: 3955.

[14] Gentry S, Gentry B. Chronic obstructive pulmonary disease: diagnosis and management. American Family Physician. 2017; 95: 433-441.

[15] Pauwels R, Buıst AS, Ma P, Jenkıns C, Hurd S; GOLD Scientific Committee. Global strategy for the diagnosis, management, and prevention of chronic obstructive pulmonary disease: National Heart, Lung, and Blood Institute and World Health Organization Global Initiative for Chronic Obstructive Lung Disease (GOLD): executive summary. American Journal of Respiratory and Critical Care Medicine. 2001; 46: 798-825.

[16] Kranenburg AR, Willems-Widyastuti A, Mooi WJ, Sterk PJ, Alagappan VKT, de Boer WI, et al. Enhanced bronchial expression of extracellular matrix proteins in chronic obstructive pulmonary disease. American Journal of Clinical Pathology. 2006; 126: 725-735.

[17] Prabhala P, Wright DB, Robbe P, Bitter C, Pera T, ten Hacken NHT, et al. Laminin $\alpha 4$ contributes to airway remodeling and inflammation in asthma. American Journal of Physiology-Lung Cellular and Molecular Physiology. 2019; 317: L768-L777.

[18] Liesker JJ, Ten Hacken NH, Zeinstra-Smith M, Rutgers SR, Postma DS, Timens W. Reticular basement membrane in asthma and COPD: similar thickness, yet different composition. International Journal of Chronic Obstructive Pulmonary Disease. 2009; 4: 127-135.

[19] Westerhof GA, Korevaar DA, Amelink M, de Nijs SB, de Groot JC, Wang $\mathrm{J}$, et al. Biomarkers to identify sputum eosinophilia in different adult asthma phenotypes. European Respiratory Journal. 2015; 46: 688-696.

[20] Brusselle G, Pavord ID, Landis S, Pascoe S, Lettis S, Morjaria N, et al. Blood eosinophil levels as a biomarker in COPD. Respiratory Medicine. 2018; 138: 21-31.

[21] Tashkin DP, Wechsler ME. Role of eosinophils in airway inflammation of chronic obstructive pulmonary disease. International Journal of Chronic Obstructive Pulmonary Disease. 2018; 13: 335-349.

[22] Hospers JJ, Schouten JP, Weiss ST, Postma DS, Rijcken B. Eosinophilia is associated with increased all-cause mortality after a follow-up of 30 years in a general population sample. Epidemiology. 2000; 11: 261-268.

[23] Nixon J, Newbold P, Mustelin T, Anderson GP, Kolbeck R. Monoclonal antibody therapy for the treatment of asthma and chronic obstructive pulmonary disease with eosinophilic inflammation. Pharmacology \& Therapeutics. 2017; 169: 57-77.

[24] George L, Brightling CE. Eosinophilic airway inflammation: role in asthma and chronic obstructive pulmonary disease. Therapeutic Advances in Chronic Disease. 2016; 7: 34-51.

[25] Casanova C, Celli BR, de-Torres JP, Martínez-Gonzalez C, Cosio BG, Pinto-Plata V, et al. Prevalence of persistent blood eosinophilia: relation to outcomes in patients with COPD. European Respiratory Journal. 2017; 50: 1701162 .

How to cite this article: Gulay Dasdemir Ilkhan, Hakan Celikhisar. Serum laminin levels in eosinophilic and noneosinophilic chronic obstructive pulmonary disease patients. Signa Vitae. 2021;17(2):188-192. doi:10.22514/sv.2021.018. 